\title{
Non-invasive blood glucose measurement by near infrared spectroscopy: Machine drift, time drift and physiological effect
}

\author{
Simon C.H. Lam*, Joanne W.Y. Chung, K.L. Fan and Thomas K.S. Wong \\ School of Nursing, The Hong Kong Polytechnic University, Hong Kong, China
}

\begin{abstract}
The aim of this paper is to evaluate development of the non-invasive blood glucose measurement of near infrared (NIR) spectroscopy. The results showed that NIR spectroscopy might obtain glucose concentration of up to $200 \%$ difference under a same environmental condition with two months apart due to time and machine drifts. These effects can restrict the development of the non-invasive blood glucose measurement. Partial least square (PLS) regression was used, which showed advantage over using simple absorbance for glucose concentration. Non-invasive blood glucose measurement of health subjects (non-diabetics) was also investigated. The results showed that $\mathrm{R}$ correlation coefficient of prediction $\left(R_{\mathrm{p}}\right)$ was 0.48 and root mean square of prediction (rmsep) was $1.34 \mathrm{mmol} / \mathrm{l}$. The error was mainly due to the physiological effect of different subjects.
\end{abstract}

Keywords: Spectroscopy, machine drift, time drift, physiological effect, PLS, PDS, Beer's law

\section{Introduction}

Diabetes mellitus (DM) is a chronic and incurable disease caused by the malfunction of insulin production which affects the blood glucose $[1,8,10]$ in the body, normally leading to hyperglycemia. Prolonged hyperglycemia causes damage to nerves and blood vessels $[1,8,10]$, which may cause many complications. Thus, the role of blood glucose monitoring and measurement becomes very important for diabetic therapies.

\subsection{Diabetes}

The number of diabetics is increasing. According to World Health Organization (WHO), there were 171 million people with DM worldwide [6,11]. In the year 2030, the number of diabetics may reach to 366 million, of which 90-95\% will suffer from Type 2 diabetes [6,9]. The highest prevalence was in the developing countries, India and China (20.8 million) in the year 2000 [6]. It is predicted that the number of the diabetics will significantly increase to 42.3 million in China after 30 years [6].

Finger-prick glucose measurements are common and can provide reliable glucose readings. They do not continuously monitor blood glucose and therefore cannot present a precise glucose trend. In addition, since blood samples are needed for the glucose measurement, this repeated sampling causes pain and damage to the skin tissue, which may raise the chance of infection.

\footnotetext{
* Corresponding author: Simon C.H. Lam, School of Nursing, The Hong Kong Polytechnic University, Hong Kong, China. Tel.: +852 8210 0441; Fax: +852 8210 0517; E-mail: simon-lam@live.com.
} 
A non-invasive approach is the painless method for blood glucose measurement. However, no noninvasive blood glucose meter currently in use has provided consistently reliable results, due to many interfering non-specific physiological signals during the measurement (especially for long-term measurement). According to Danzer et al. [3], the long-term unreliability might be due to the physiological differences amongst subjects and time drift factors. Thus, diabetic patients still need to rely on painful finger-prick sampling for daily glucose measurement.

\section{Experiment and clinical trial}

An experiment by using glucose solutions (see Fig. 1) and a clinical trial with healthy subjects had been conducted.

\subsection{Glucose solutions}

Control Development's NIR spectrometer of NIR-128L-1.7-USB, with the wavelength range of 905$1701 \mathrm{~nm}$ was used. D-Glucose dissolved in deionised water was used for preparing different glucose concentrations. A thermo-resistant petri dish made of polystyrene (PS) was used as the container because of its high temperature resistance and good optical clarity.

The known concentrations of solutions were measured by NIR spectrometer in a room with a temperature range of $24-25^{\circ} \mathrm{C}$ and a relative humidity of 55-60\%. Four sets of glucose solution measurements, each set with five concentrations $(0,125250,500$ and $1000 \mathrm{mmol} / \mathrm{l})$, were measured on three separate days by NIR spectroscopy. The average of the NIR spectra was recorded from 64 readings per sample. Calibration against the background and the reference (under tungsten halogen light) was carried before each glucose measurement.

\subsection{Clinical trial}

Thirty-six healthy subjects voluntarily joined the clinical trial and had been fasting overnight before the first NIR and the first finger-prick glucose measurement in the morning. The subject group comprised eight males and 28 females, all of whom were non-diabetic.

Finger pricks and NIR measurements were performed at the preprandial (fasting stage) and again at the postprandial (after had breakfast) stage. The whole procedure for data capture (including NIR spectra, finger surface temperature and finger-prick glucose levels) took approximately 3-5 min, per patient. Each subject was provided the same breakfast at the same quantity after the preprandial measurement. A sucrose candy had also been provided for each subject 15-30 min before the postprandial measurements. This helped to increase their respective glucose level, thus the glucose levels would be varied.

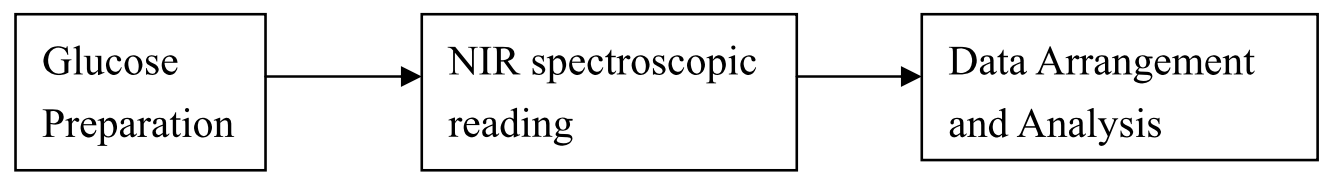

Fig. 1. Block diagram - glucose concentration analysis. 


\section{Analytical methods}

According to Beer's law, the absorbance is related to the concentration. Thus, based on Beer's law, a single wavelength was selected for the glucose concentration evaluation. Another technique of partial least square (PLS) prediction [4,7] was also applied for the analysis. One group of data from two different experiments were used for calibrations, another one was for prediction and validation purposed as shown in Fig. 2.

The spectra obtained were separated into two groups. One group (Spectra A) was used for calibration with the corresponding glucose concentrations and formed the calibration model (Model) shown on Eqs (1) and (2).

$$
g_{1}=M_{1} X_{1}+e_{1},
$$

where $X_{1}-$ Spectra A, $e_{1}$ - error $1, g_{1}$ - corresponding glucose concentrations, $M_{1}$ - calibration model built.

Then, Spectra B were substituted to $M_{1}$ by PLS.

$$
g=M_{1} X_{2}+e_{2}
$$

where $X_{2}$ - Spectra B, $e_{2}$ - error $2, g$ - predicted glucose concentrations.

The predicted glucose concentrations $g$ were then validated with $g_{2}$, where $g_{2}$ was the glucose concentration corresponding to Spectra B.

\section{Results and discussions}

\subsection{Glucose solutions}

The results showed that pure water provided a higher absorbance spectrum. Lower absorbance spectra represented higher glucose concentrations as shown in Fig. 3. The wavelength $1180 \mathrm{~nm}$ was chosen for the analysis, as it showed the most clearly distinguished absorbance and was also located in the region of second overtones [5].

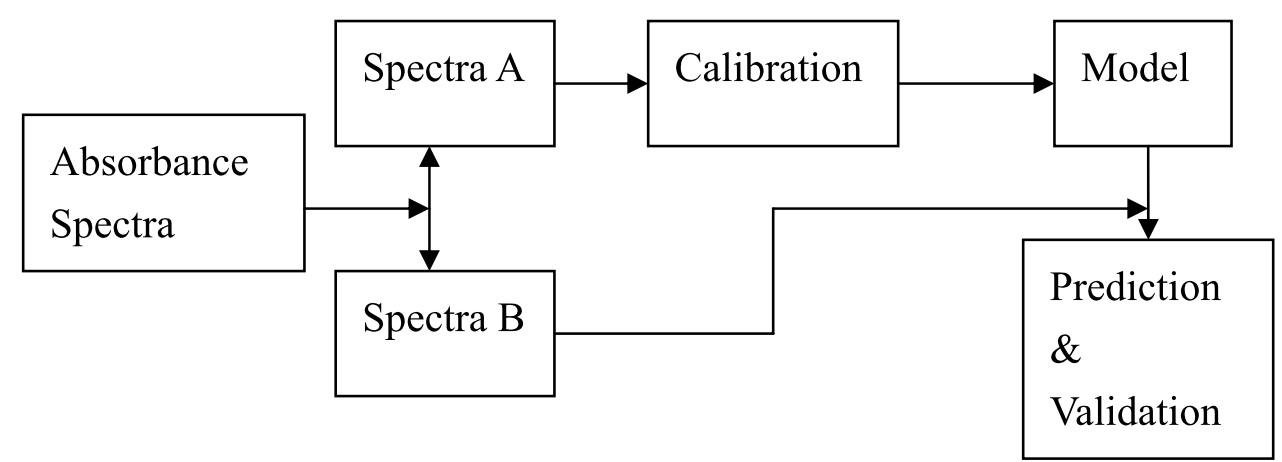

Fig. 2. Block diagram - PLS prediction process of glucose solution concentration. 


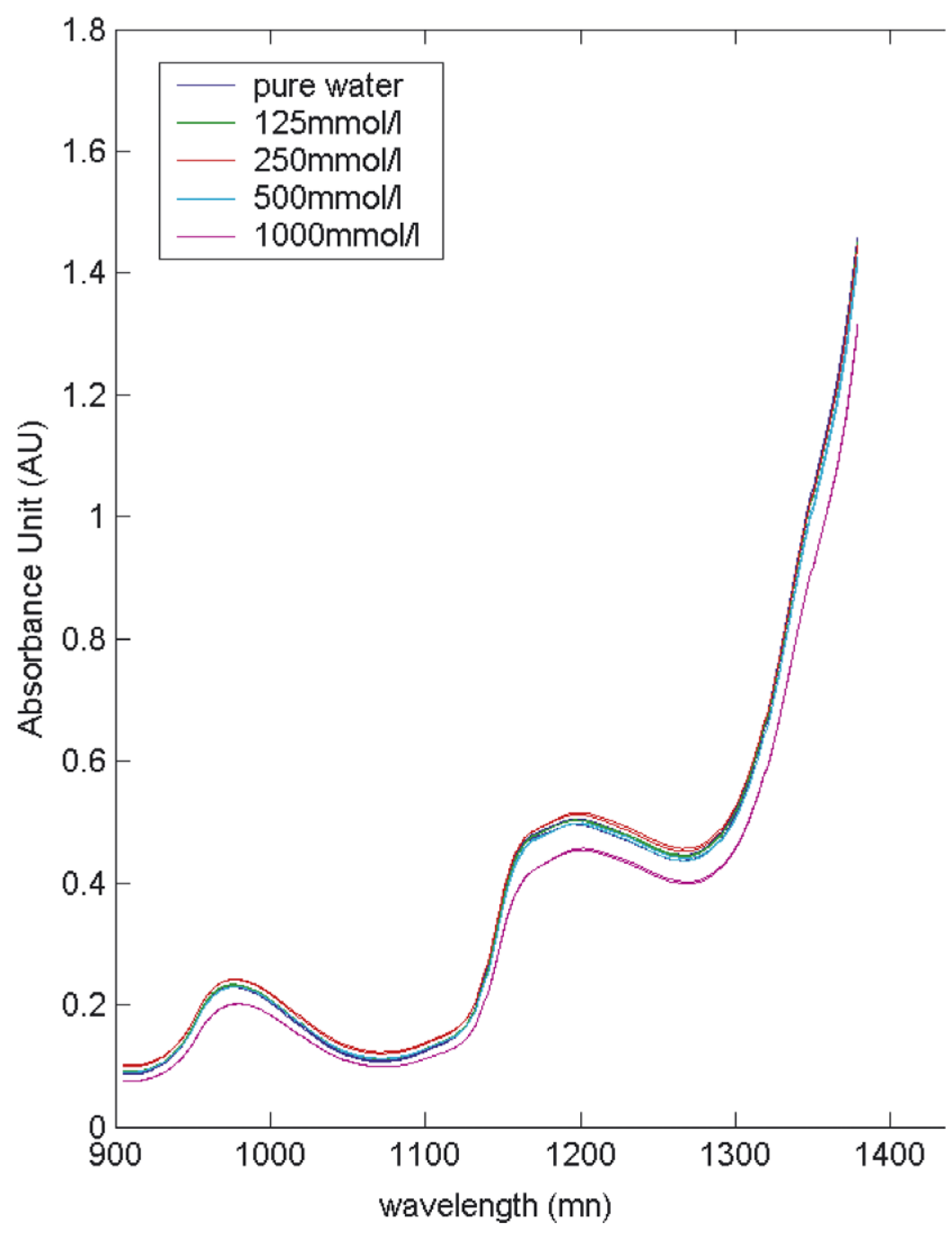

Fig. 3. NIR spectra - glucose solutions, absorbance against wavelength $(\mathrm{nm})$. Day 1 under room condition. (Colors are visible in the online version of the article; http://dx.doi.org/10.3233/SPE-2010-0485.)

\subsection{Glucose solutions measurement on the same day}

Different batches of glucose solutions were measured within one hour by NIR spectroscopy. These measurements were used to check the reliability of the NIR measurement within a short period of time, while trying to avoid machine drift and time drift. The glucose concentration was first plotted against the NIR absorbance spectra.

\subsection{Single wavelength absorbance vs. glucose concentration}

Inverse linearity was observed with absorbance spectra at wavelength $1180 \mathrm{~nm}$, where the lower absorbance indicated a higher glucose concentration. The best fitted lines, shown in Fig. 4, can be represented by the following linear equations, respectively. 


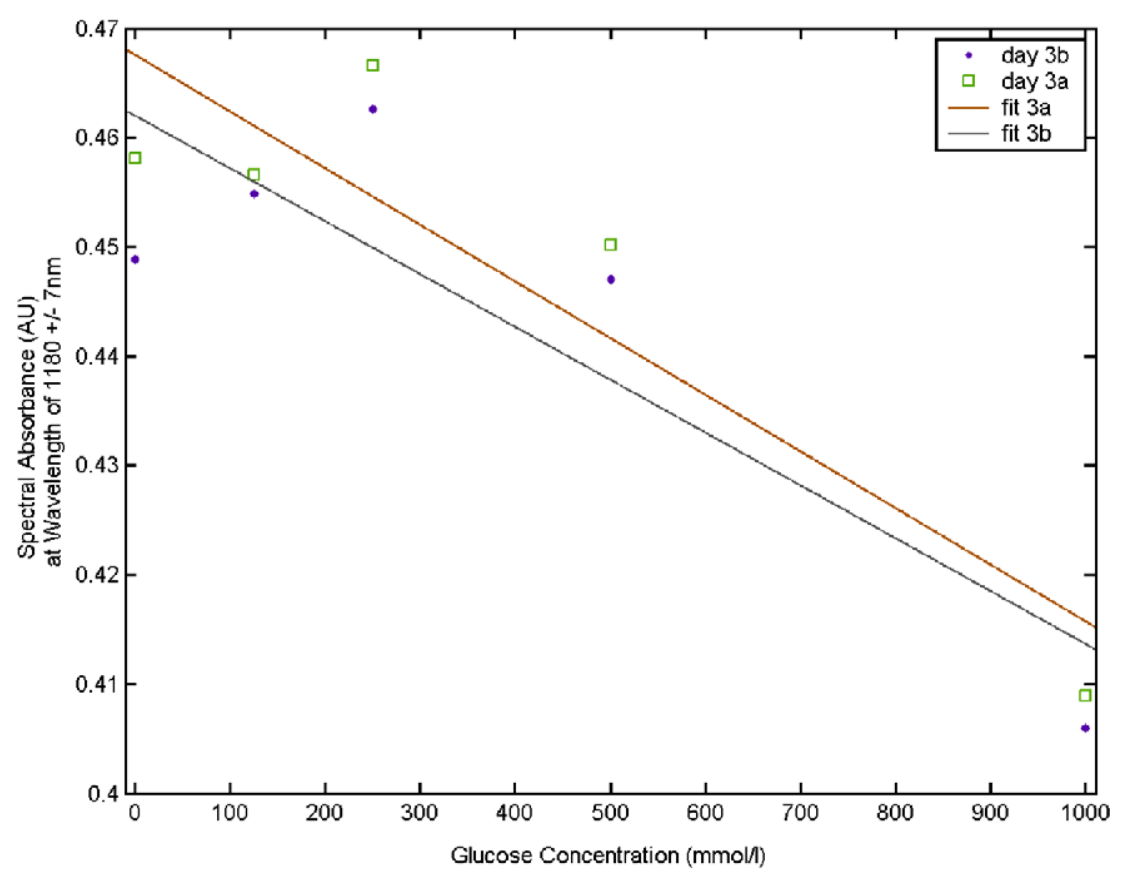

Fig. 4. Absorbance at $1180 \mathrm{~nm}$ against glucose concentration, separated experiment in one hour; fit of Day 3a is close to fit of Day 3b. (Colors are visible in the online version of the article; http://dx.doi.org/10.3233/SPE-2010-0485.)

Fit 3a:

$$
y=-5.19 \mathrm{e}^{-5} x+0.47 \quad(R=0.91 \text { and } \mathrm{RMSE}=0.0108 \mathrm{AU} \text { or } 207 \mathrm{mmol} / \mathrm{l}) .
$$

Fit 3b:

$$
y=-4.84 \mathrm{e}^{-5} x+0.46 \quad(R=0.87 \text { and RMSE }=0.0098 \mathrm{AU} \text { or } 204 \mathrm{mmol} / \mathrm{l}),
$$

where $y$ - absorbance units, $x$ - glucose concentrations, $R$ - R correlation coefficient, RMSE - root mean square error.

Figure 4 showed that the fit of $3 \mathrm{a}$ and $3 \mathrm{~b}$ were close to each other. This showed that the results of the two experiments were nearly repeated. This also demonstrated that glucose concentrations could be measured via transmittance NIR spectroscopy, where the absorbance (AU) is related to the glucose concentrations.

\subsection{Glucose concentration predictions by PLS}

PLS is another prediction technique mainly used for spectroscopic application. The spectral results were analyzed by PLS. The known glucose concentrations were calibrated with the measured spectra from Day 3a to form a model and then the measured spectra of Day $3 \mathrm{~b}$ (after one hour) were predicted through PLS. Figure 5 shows the scatter plot of the calibration results and the prediction results of the glucose concentration within one hour.

These results showed the $\mathrm{R}$ correlation coefficient of the prediction $R_{\mathrm{p}}=0.92$ and root mean square error rmse $=158 \mathrm{mmol} / \mathrm{l}$. The relationship between spectral signals (or the absorbance unit under 


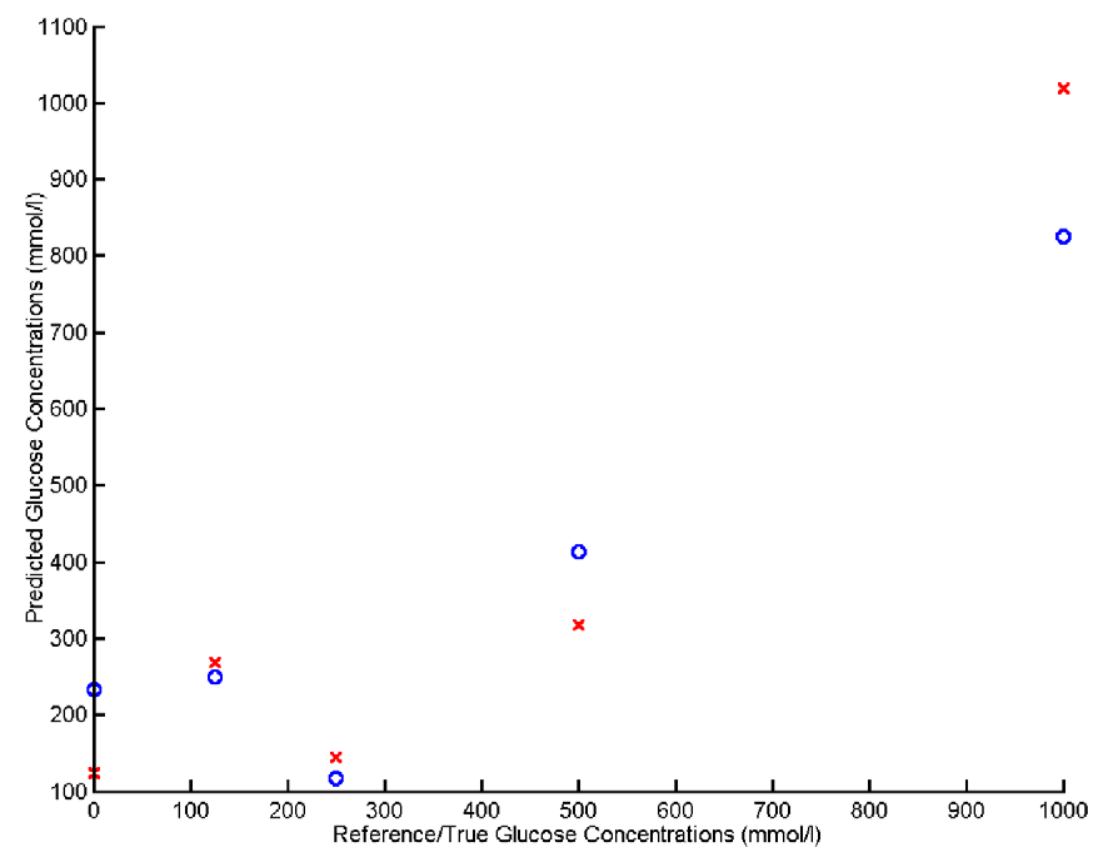

Fig. 5. PLS prediction scatter plot, separated experiment in the same day within one hour; cross $(\times)$ - calibration; hollow (o) prediction. (Colors are visible in the online version of the article; http://dx.doi.org/10.3233/SPE-2010-0485.)

tungsten halogen at $1180 \mathrm{~nm}$ wavelength) and the glucose concentration was linear. The major predicted error occurred in the low glucose range.

PLS provides another prediction technique for the glucose concentrations by using NIR spectroscopy. The poorest prediction was located in the pure water region. PLS prediction provided partly poorer results when compared with the direct absorbance to the glucose concentration analysis. However, the prediction made through PLS was still better than just using the absorbance unit. Although glucose concentration is proportional to the NIR absorbance of a single wavelength, the rate of error may be higher when only a single wavelength is used. Alternatively, PLS was applied by using the whole range of the spectra for the prediction. The error may be relatively small since used whole range spectra may cause to average the error.

\subsection{Glucose solution measurements on different days}

Different batches of known glucose solutions were measured on different days (two month apart) by the same NIR spectrometer. The glucose concentration was plotted against NIR absorbance for the evaluation. It was also analyzed by PLS.

\subsection{Single wavelength absorbance versus glucose concentration on separate days}

Absorbance of the single wavelength at $1180 \mathrm{~nm}$ was plotted against the corresponding glucose concentration, as shown in Fig. 6. The results showed that the data of Day 1 is about 0.06 AU (potentially a very significant error) higher than the data of Day 2 . Their fitting equations are shown below.

Fit 1:

$$
y=-3.32 \mathrm{e}^{-5} x+0.47 \quad(R=0.99 \text { and } \mathrm{RMSE}=0.0020 \mathrm{AU} \text { or } 60 \mathrm{mmol} / \mathrm{l}) .
$$




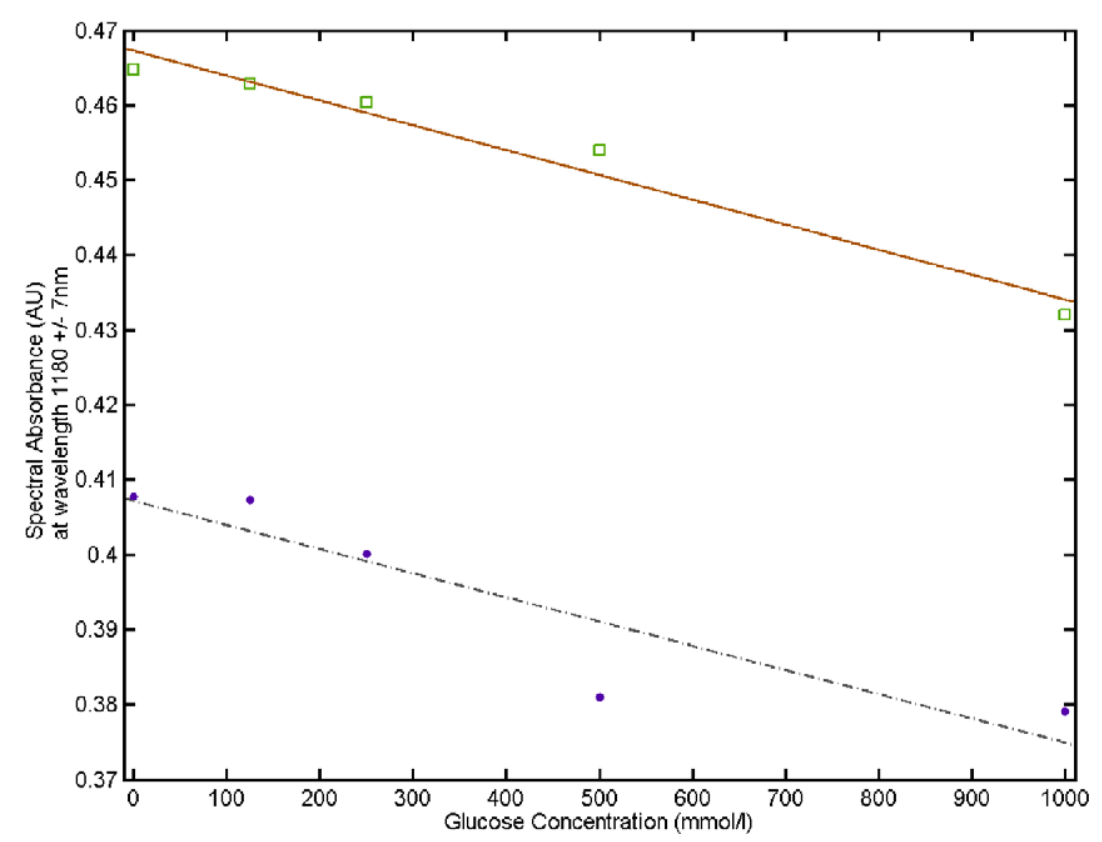

Fig. 6. Absorbance at $1180 \mathrm{~nm}$ against glucose concentration, two experiments in two months separation (with two preparations for same concentrations). (Colors are visible in the online version of the article; http://dx.doi.org/10.3233/SPE-2010-0485.)

Fit 2:

$$
y=-3.23 \mathrm{e}^{-5} x+0.41 \quad(R=0.91 \text { and } \mathrm{RMSE}=0.0052 \mathrm{AU} \text { or } 163 \mathrm{mmol} / \mathrm{l}),
$$

where $y$ - absorbance units, $x$ - glucose concentrations, $R$ - R correlation coefficient, RMSE - root mean square error.

$0.06 \mathrm{AU}$ represents $2000 \mathrm{mmol} / \mathrm{l}$. This showed that the results might not be reproducible under a single wavelength measurement after two months. This was due to machine drift and time drift. Even though there was only one variable - glucose, the machine might face micro-deviation among the different inner parts which accumulated over a period of time.

\subsection{Spectra prediction versus glucose concentration by PLS on separate days}

Preprocess of Piecewise Direct Standardization (PDS) was used to regulate the spectral drifts from different days. These preprocessed data were then calibrated and predicted by PLS regression. The results were then scatter plotted, as shown in Fig. 7.

The results showed that $R_{\mathrm{p}}=0.97$ and rmsep $=144 \mathrm{mmol} / \mathrm{l}$. A major error also occurred in the low glucose concentration region, particularly for the pure water measurement. Table 1 shows the summary of $R$ and rmse ( $R_{\mathrm{p}}$ and rmsep) by absorbance and PLS spectral analysis, respectively.

\section{Error and noise}

There were possible measurement errors, or noises, of the glucose solution. The experiment was carried out under the exposure to the surrounding environment. The results might not be affected by the 


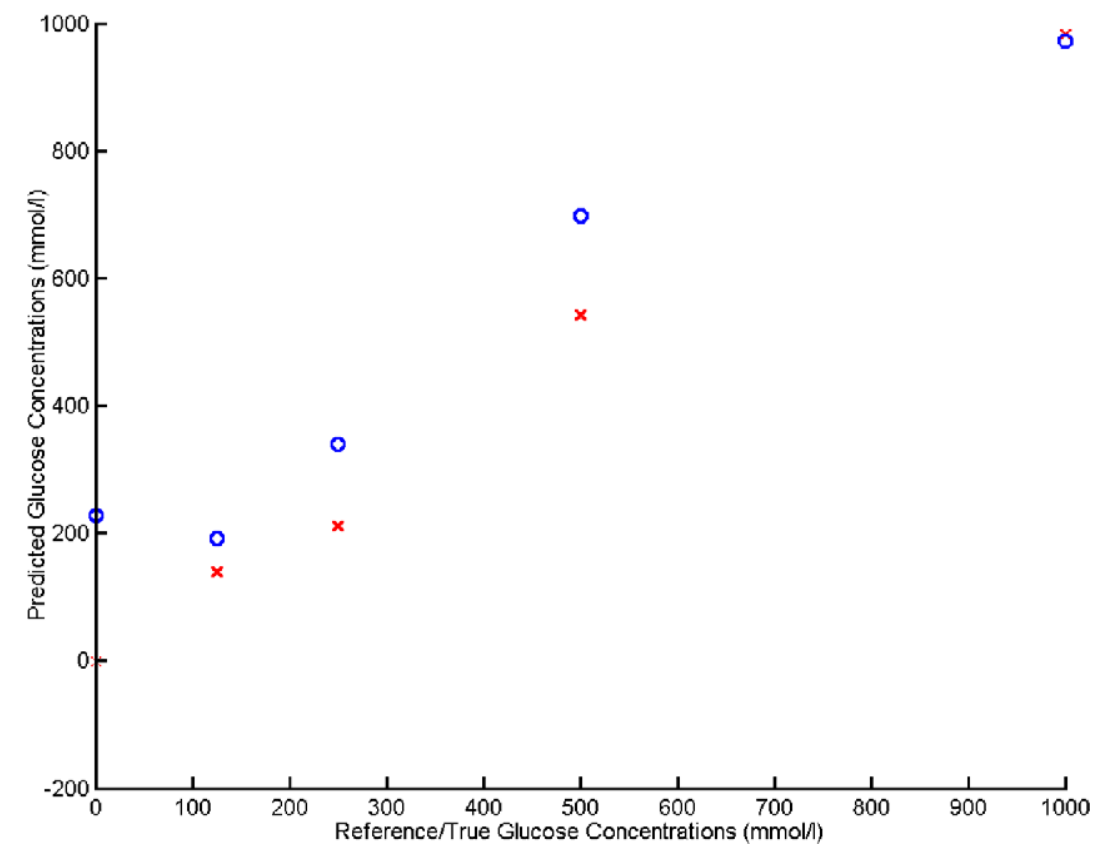

Fig. 7. PLS prediction scattered plot, separated experiment in different days in two month: cross $(\times)$ - calibration; hollow (o) prediction. (Colors are visible in the online version of the article; http://dx.doi.org/10.3233/SPE-2010-0485.)

Table 1

$\mathrm{R}$ correlation of coefficient and rmse (root mean square error) comparison; absorbance of Day $1>0.06 \mathrm{AU}$ of absorbance of Day 2

\begin{tabular}{|c|c|c|c|c|}
\hline \multirow[t]{3}{*}{ Analysis } & \multicolumn{4}{|c|}{ Day } \\
\hline & \multicolumn{2}{|c|}{ Under same day } & \multicolumn{2}{|c|}{ Under different days } \\
\hline & $R$ & RMSE (mmol/l) & $R$ & $\operatorname{RMSE}(\mathrm{mmol} / \mathrm{l})$ \\
\hline \multirow[t]{2}{*}{ Absorbance } & 0.91 & 207 & 0.99 (Day 1) & 60 (Day 1) \\
\hline & 0.87 & 204 & 0.91 (Day 2) & 163 (Day 2) \\
\hline PLS (prediction) & $R_{\mathrm{p}}=0.92$ & rmsep $=158$ & $R_{\mathrm{p}}=0.97$ & rmsep $=144$ \\
\hline
\end{tabular}

surrounding florescent light which exists at $190 \mathrm{~nm}$ to $650 \mathrm{~nm}$, while NIR is ranged from $730 \mathrm{~nm}$ to $2500 \mathrm{~nm}$. The indium gallium arsenide (InGaAs) detector (NIR detector could only sense within NIR region) of the spectrometer was not affected by the surrounding visible light. However, NIR might have derived from other NIR environmental sources, such as temperature variations, if some of the other equipment present generated an NIR wavelength range.

In addition, the resolution of the spectrometer is $6.22 \mathrm{~nm}$, thus, the specificity of the absorbance at $1180 \mathrm{~nm}$ could be $1180 \pm 6.22 \mathrm{~nm}$. The results might not really represent the absorbance of the exact corresponding wavelength. However, NIR overtones always overlaps to multiple wavelengths or a certain range of the spectrum (83). The separation of each measurement pixel forms the spectrum by interpolation. This indicates that the resolution might not provide a significant effect to the absorbance. Unless there was a particular wavelength that had fallen in between two pixels, error might happen. However, as NIR overtones always cover multiple wavelengths, the resolution of the absorbance was enough to cover the measurement. 
Nevertheless, the measurement area in the experiment was relatively large within the petri dish, and it might affect the results, particularly on the low glucose concentration. The glucose particles might not be equally distributed among the large area. The error could be relatively large due to the outside environment and the glucose preparation error, particularly in low glucose concentration areas.

\subsection{Clinical trial}

A total of 108 sets of spectra were obtained. Thirty-six sets of spectra were obtained before breakfast. Seventy-two sets of spectra (where one additional set of spectra was obtained from each subject in postprandial measurement) were obtained after breakfast. Figure 8 shows a spectral plot of absorbance against wavelength. The spectra were similar to those shown in Fig. 3. Overtones also occurred on $980 \mathrm{~nm}$ and $1200 \mathrm{~nm}$. PLS was applied for the prediction (each third of the data of the spectra and the corresponding glucose readings were used for prediction, while the remaining sets of data were used for calibration). Results are presented in the scatter plot shown in Fig. 9.

Figure 9 showed that both the calibrations and predictions were located within the clinical, acceptable regions, $\mathrm{A}$ and $\mathrm{B}$. The $\mathrm{R}$ correlation coefficient of the calibration $\left(R_{\mathrm{c}}\right)$ was 0.85 and the root mean square

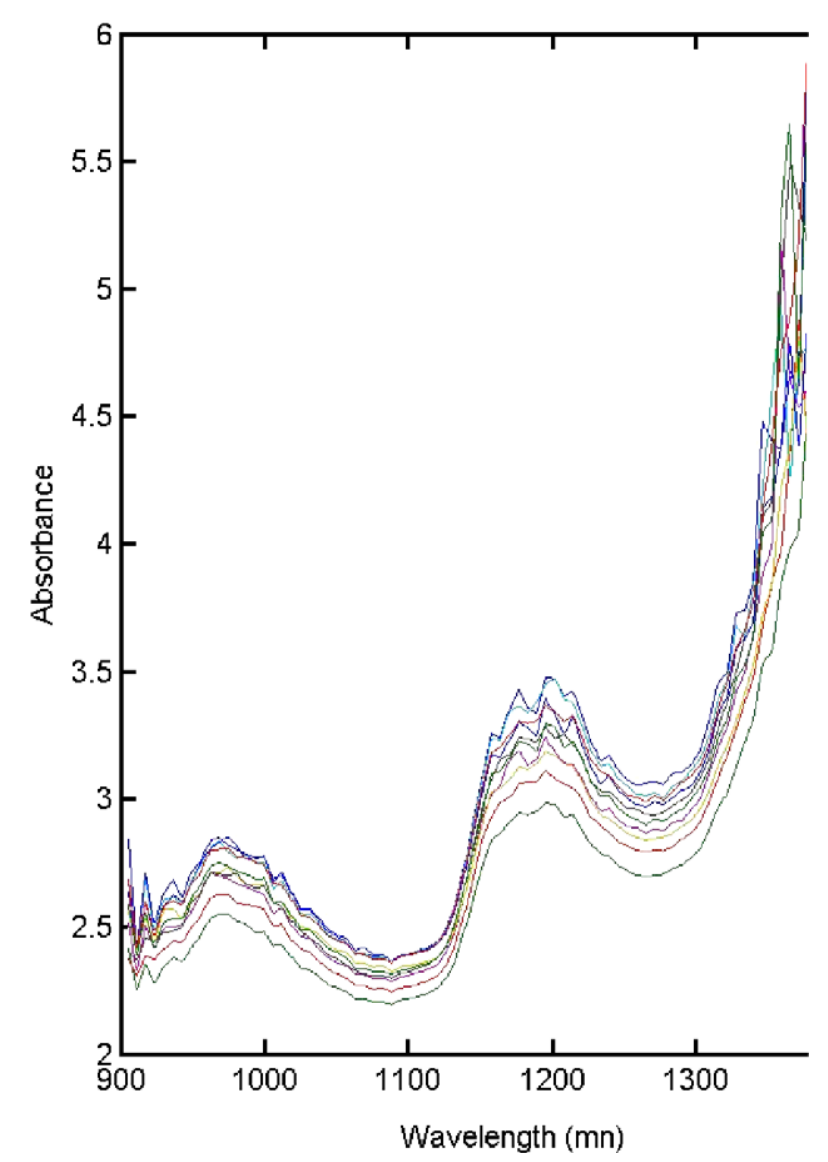

Fig. 8. Absorbance against wavelength, healthy subject. (Colors are visible in the online version of the article; http://dx.doi.org/ 10.3233/SPE-2010-0485.) 


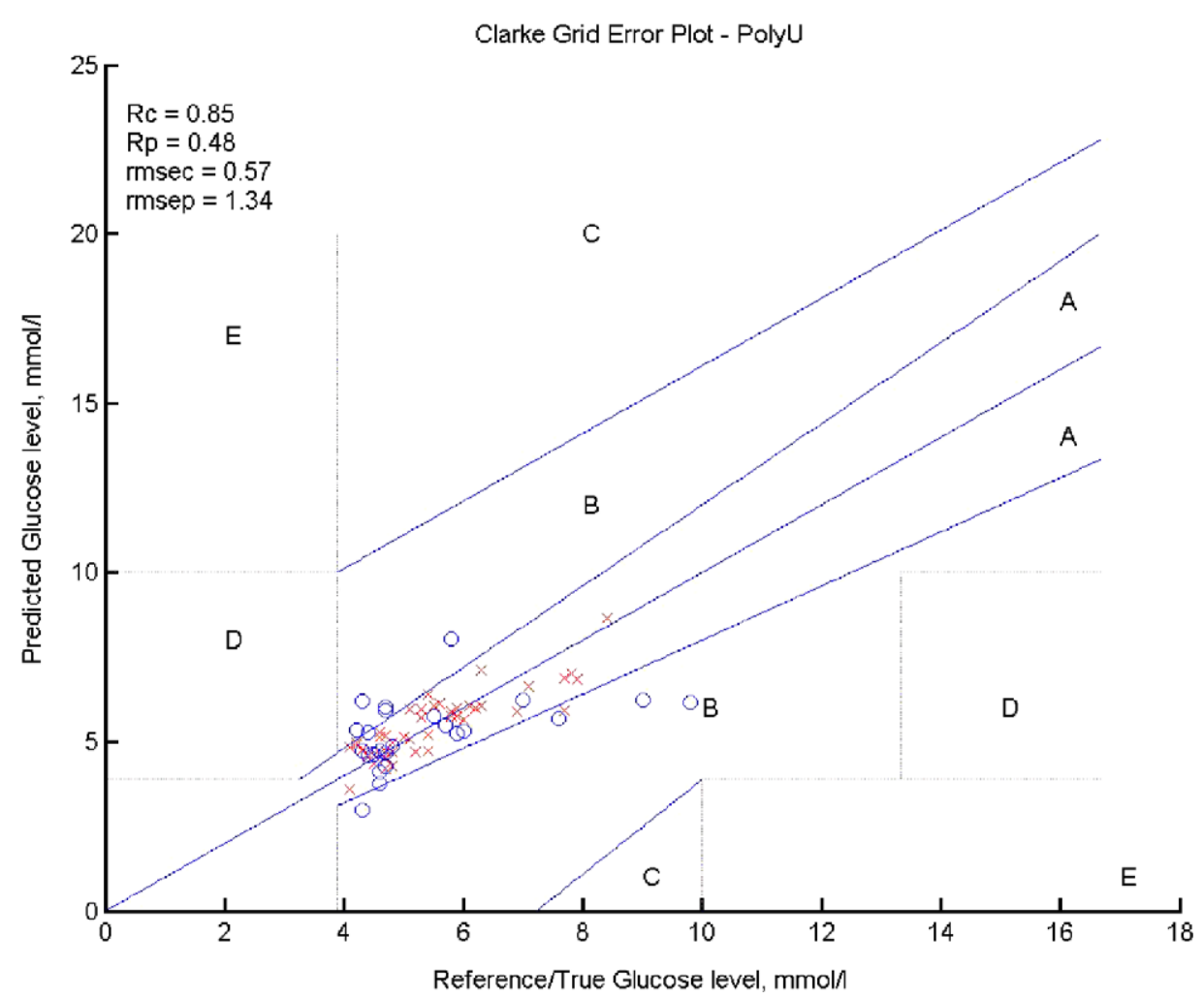

Fig. 9. Scatter plot of predicted against finger-prick blood glucose level with simple mean-centred preprocess and PDS preprocess, for full wavelength and non-arranged data: $(\times)$ - calibration, $(\circ)$ - prediction. (Colors are visible in the online version of the article; http://dx.doi.org/10.3233/SPE-2010-0485.)

error of calibrations (rmsec) was $0.57 \mathrm{mmol} / \mathrm{l}$. The $\mathrm{R}$ correlation coefficient of the prediction $\left(R_{\mathrm{p}}\right)$ was 0.48. The root mean square error of prediction (rmsep) was $1.34 \mathrm{mmol} / \mathrm{l}$.

Most of the prediction spots fell in Region A, where errors were within 20\%. Regions A and B were clinically acceptable regions as stated by Clarke Grid Error plot [2]. This demonstrated that NIR spectra and the blood glucose levels of non-diabetics were correlated after applying PLS for the prediction.

The results showed that the study of non-diabetics provided a positive sign for non-invasive blood glucose prediction. However, high variation as shown in Fig. 8 may lead to difficulties for blood glucose prediction. The spectral variation might indicate that higher noises or uncertainty of signals might be obtained in addition to the necessary signals. This might be due to time drift, machine drift and physiological differences in a long-term clinical measurement.

The physiological influence from the human body was one of the key effects in relation to non-invasive blood glucose measurement. Time factors have led to uncertain physiology, which could affect the prediction. The longer time span between each measurement, the more uncertainties due to the physiology occur; thus, the poorer the calibration and the prediction obtained. This is because of the higher chance of the reaction of the human body to both internal and external environmental changes. Therefore, the study of healthy subjects in short-term might have provided better predictions due to the shorter time span and relatively fewer physiological uncertainties. However, $R_{\mathrm{p}}$ and rmsep were 0.48 and $1.34 \mathrm{mmol} / \mathrm{l}$, respectively. This was because of physiological uncertainties between subjects. 
These two studies showed that 'time and machine drifts' and 'physiological effect' could affect in vivo blood glucose prediction. However, as mentioned, the glucose solution experiment showed that machine drift and time drift could be reduced by using PLS with preprocess. Thus, the error might be due to physiological differences between subjects.

Furthermore, the results of this clinical trial also showed that the larger variation caused larger error for PLS predictions. The large variation might have been due to the physiological influences, including temperature. Thus, NIR measurement also needed to be improved by reducing the variation during the measurements.

\section{Conclusions}

Results showed that higher glucose concentration, the lower the spectral absorbance was in the spectrum. However, NIR spectroscopic measurements were deviated due to machine drift and the time drift after two months. This caused errors in the NIR spectroscopic measurements. These drifts could be reduced or eliminated by PLS technique. NIR spectroscopic measurement under PLS analysis showed promising results on the glucose solution test in both short- and long-term experiments.

However, physiological effects are unpredictable and varied from time to time due to the human body's reaction to the environment. This led to the most uncertainty during the clinical trial. Thus, considering the physiological influence for NIR non-invasive blood glucose measurements would be needed.

\section{References}

[1] ADA, All about diabetes, American Diabetes Association, 2006, available at: http://www.diabetes.org/about-diabetes.jsp.

[2] W.L. Clarke, D. Cox, L.A. Gonder-Frederick, W. Carter and S.L. Pohl, Evaluating clinical accuracy of systems for selfmonitoring of blood glucose, Diabetes Care 10 (1987), 622-628.

[3] K. Danzer, C. Fischbacher, K.U. Jagemann and K.J. Reichelt, Near-infrared diffuse reflection spectroscopy for noninvasive blood-glucose monitoring, IEEE 12(2) (1998), available at: http://www.iee.org/organizations/pubs/newsletters/ leos/apr98/monitoring.htm.

[4] S. de Jong, SIMPLS: An alternative approach to partial least squares regression, Chemometrics and Intelligent Laboratory Systems 18 (1993), 251-263.

[5] B. Jorgensen and Y. Goegebeur, Multivariate Data Analysis and Chemometrics, Department of Statistic, University of Southern Denmark, Denmark, 2006.

[6] W. Sarah, R. Sicree, G. Roglic, H. King and A. Green, Global prevalence of diabetes: Estimates for the year 2000 and projections for 2030, Diabetes Care 27 (2004), 1047-1053.

[7] R.D. Tobias, An introduction to partial least squares regression, SAS Institute Inc., Cary, NC, USA, 1998, available at: http://support.sas.com/rnd/app/papers/pls.pdf.

[8] WHO, Diabetes mellitus, World Health Organization Media Center, April 2002, Fact Sheet No. 138.

[9] WHO, Screening for type 2 diabetes, World Health Organization, 2003.

[10] WHO, Diabetes programme: What is diabetes?, World Health Organization, 2006, available at: http://www.who.int/ diabetes/BOOKLET_HTML/en/index4.html.

[11] WHO, Diabetes programme: A rising global burden, World Health Organization, 2006. 


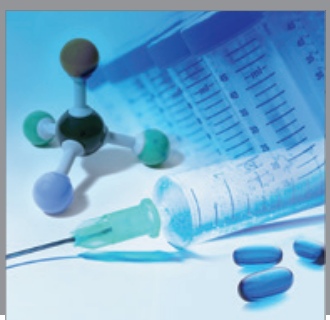

International Journal of

Medicinal Chemistry

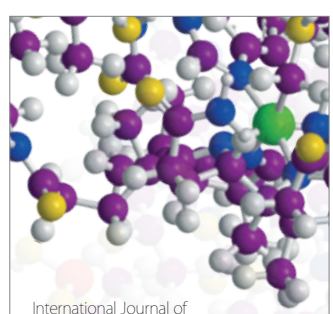

Carbohydrate Chemistry

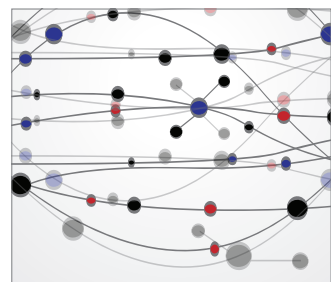

The Scientific World Journal
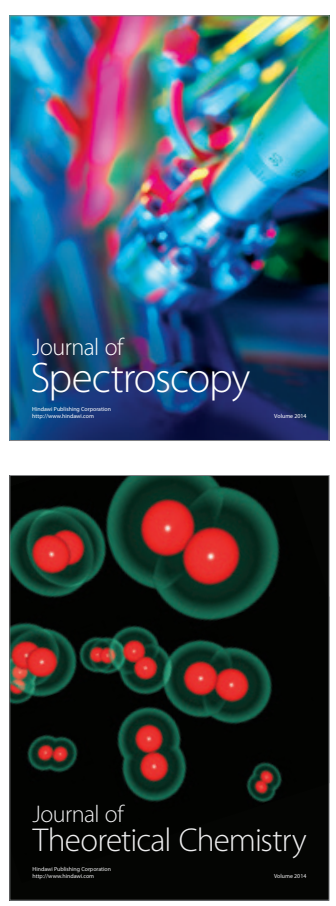
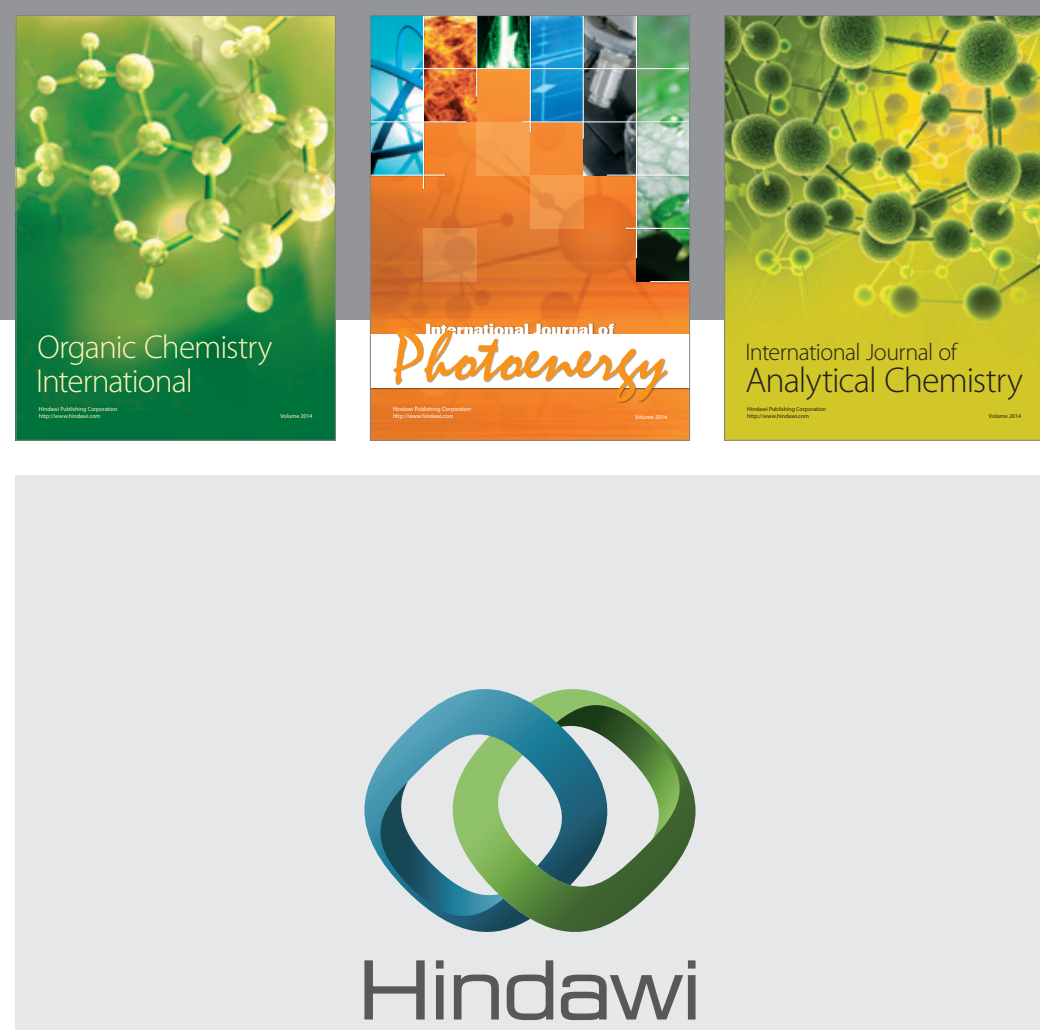

Submit your manuscripts at

http://www.hindawi.com
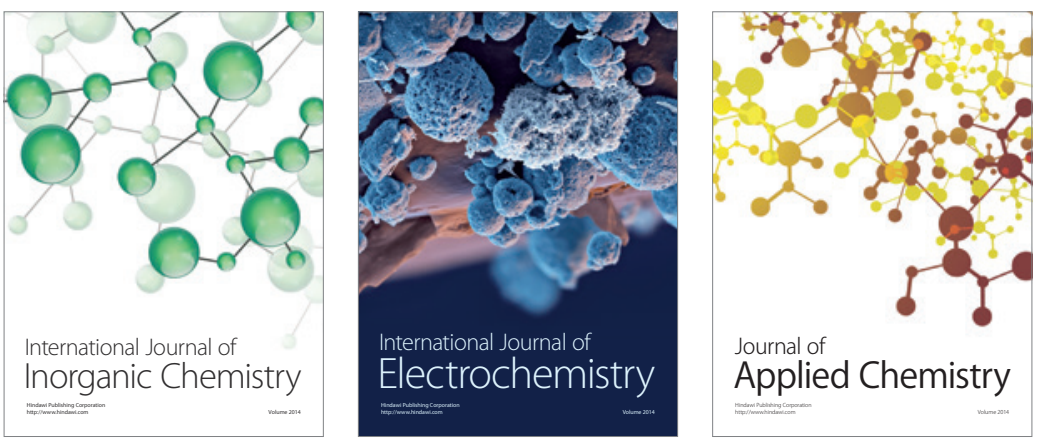

Journal of

Applied Chemistry
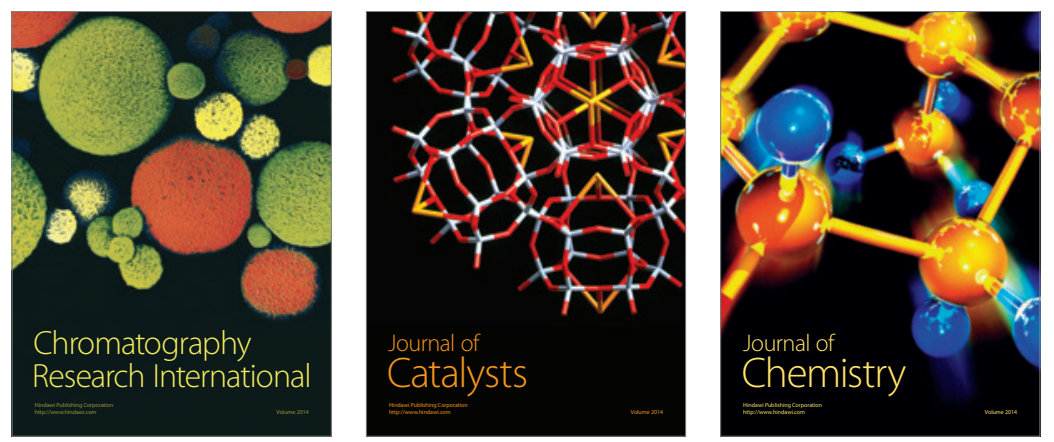
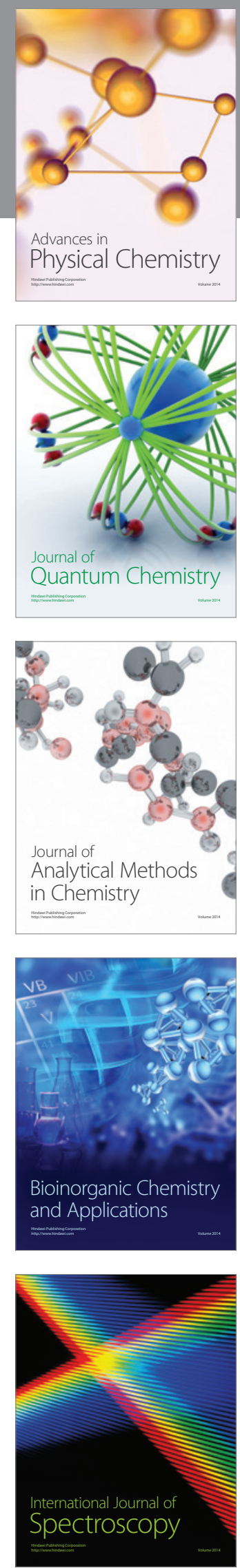